\title{
An Improved Re-Route Model to Reduce Traffic in Multi-Protocol Label Switching Network
}

\author{
Mrinal Kishor $^{1}$, Deepak Dhadwal ${ }^{2}$ \\ M.Tech Research Scholar, Electronics \& Communication Engg Department, M.M University, \\ Sadopur Ambala, Haryana, India ${ }^{1}$ \\ Assistant Professor, Electronics \& Communication Engg Department, M.M University, \\ Sadopur Ambala, Haryana, India ${ }^{2}$
}

\begin{abstract}
In this work, it presents a work on improved Rerouting model in MPLS Network for reducing traffic. This paper describes the simulation to evaluate the performance of MPLS Network. QoS is the mechanism of the network to provide different service level to a different traffic type as business need. The main objective of this work is to improve QoS in network by reducing link load and bandwidth consumption. It uses a routing scheme that satisfies expected demand and minimized link utilization of system. It works on reliability by limited usage of bandwidth. Results are presented to demonstrate the effectiveness of system. The projected mechanism is implemented with MATLAB.
\end{abstract}

Keywords: MPLS Network, Congestion Management, Re-Route Model, Quality of Service etc.

\section{INTRODUCTION}

Multiprotocol Label Switching (MPLS) networks are currently the most used transport technology for service provider networks. This has happened mainly because of the various different features available in a single solution which wasn't possible to achieve by any other transport technology used that time.

Multiprotocol Label Switching (MPLS) is a versatile solution to address the problem faced by present day network speed, scalability, Quality of Service (QoS) management and traffic engineering. MPLS has emerged as an elegant solution to meet the bandwidth management and service requirement for next generation Internet Protocol (IP) based backbone networks. It addresses issues related to scalability and routing (based on QoS and service quality metrics) and can exist over existing Asynchronous Transfer Mode (ATM) and frame-relay networks. MPLS is an Internet Engineering Task Force (IETF) specified framework that provides for the efficient designation, routing, forwarding and switching of traffic flows through the network.

The first working group of the IETF that were supposed to design MPLS and address the problems that service providers were facing that time took place in 1997. This working group still exists and since then MPLS has grown into a protocol which is widely used and dependent in many network environments. The designers initially tried to address the most problematic issues at that time with the potential for further development. They had to come up with an idea which would allow for faster routing decisions with backwards compatibility. This allows MPLS to operate above any existing layer 2 protocol with the encapsulation of network layer. It is a very strong feature which allows the interconnecting of different network technologies and grouping them under one solution. The protocol is using its own addressing scheme based on labels.
Internet Service Providers (ISP) is interested in the addition of multicast traffic distribution streams capabilities to their traffic engineered MPLS network. This would provide superior IP multicast transmission which would be enhanced with QoS awareness. This QoS is required by multimedia provision on-demands businesses. Multi-Protocol Label Switching (MPLS) is a multi-layer switching technology that uses labels to determine the method in which packets are forwarded through an MPLS network. Forwarding packets refer to the common operation that both switches and routers perform on packets of a connectionless network. This operation entails receiving packets at the input, analyzing the content of the packets' header and determining the appropriate output to be transmitted.

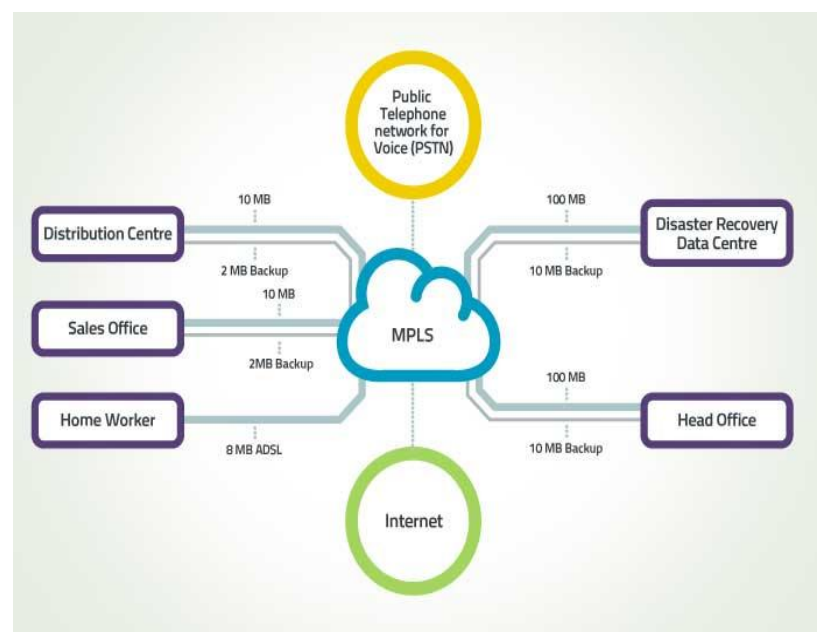

Figure 1: MPLS Network Services

The rest of paper is ordered as follows. In section II, it discusses traffic engineering with MPLS networks. In Section III, it describes proposed work plus 
implementation of system. Results are given in section V. Finally, conclusion is explained in Section V.

\section{TRAFFIC ENGINEERING}

Traffic engineering (TE) plays a key role in enabling an efficient use of the provider's resources. First, it allows the distribution of traffic in order to avoid the creation of bottlenecks. Second and taking into account the QoS requirements of the applications; TE provides the ability to route traffic on links that provide an adapted level of performance. Traffic engineering is a technique to control the flow of data over the network by reserving bandwidth for specific services. TE may be also implemented to accommodate network maintenance.

The objective of the traffic engineering technique is to improve the performance of the operational network at the resource level as well as the traffic level. Parameters such as packet loss, delay, jitter, and throughput are used to measure the network performance. To choose between different routing paths, most IP networks use Interior Gateway Protocols (IGP) based on the Open Shortest Path First (OSPF) algorithm with static link weights. These weights provide the routers with a complete view of the network to populate routing tables. When links have distinct capacities, considering link utilization is more appropriate. Network engineers employ a number of tools to automate the process of monitoring network links and to send alerts when a link is heavily used.

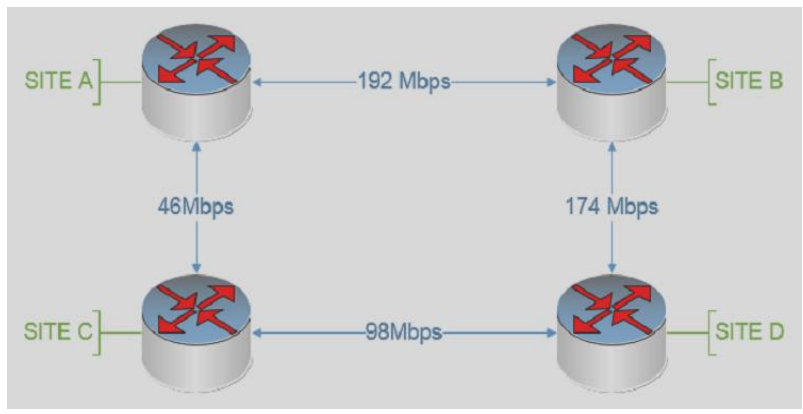

Figure 2: Traffic engineering in IP network [2]

\section{PROPOSED MPLS NETWORK SYSTEM}

MPLS can be considered a technology that has brought an oriented connection for IP protocol. Therefore, network services and applications can exploit all of the advantages of MPLS. High QoS requirement is one of the major issues for network service providers. So much work has been done in this area to improve the quality of service of the MPLS network by optimizing the QoS parameters such as bandwidth optimization, low packet loss ratio, low delay and jitter. In this, we have designed a mathematical model for the efficient use of the bandwidth required by an MPLS routing path. The effective bandwidth required by any transmission path is dependent on some factor like packet loss, transmission delay at each link etc. So effect of these parameters on bandwidth requirement is also considered in this dissertation. In this work we have presented a distributed LSP scheme to reduce the spare bandwidth demand in MPLS networks. The main idea of the proposed D-LSP scheme is to partition traffic into multiple LSPs, each of which is established on a distinct link disjoint route between each pair of end nodes.

The reason why MPLS networks have become so successful is due to the ability of implementing a multiservice network. Having a network infrastructure which consists of a variety of different technologies, while still being maintained by a single standard that provides everything ever needed to control the traffic has no competition. These two requirements impose that to support multicast, one should observe that an LSR is able to select a particular multicast distribution tree based on the following criteria. Firstly, the label carried in the packet (packet has an identity within MPLS domain) and secondly, the interface on which the packet was received.

In MPLS, transmission occurs on label-switched paths (LSPs). LSPs are a sequence of labels at each and every node along the path from source to destination. LSPs are established either prior to data transmission or upon a certain flow of data. The labels, which are underlying protocol specific-identifiers, are distributed using distribution protocol (LP) or RSVP or piggybacked on routing protocol like border gateway protocol (BGP) or OSPF. Each data packet encapsulates and carries the label during their journey from source to destination shown in figure. High speed of data is possible because fixed-length labels are inserted at the beginning of the packet and can be used by hardware to switch packets quickly between links.

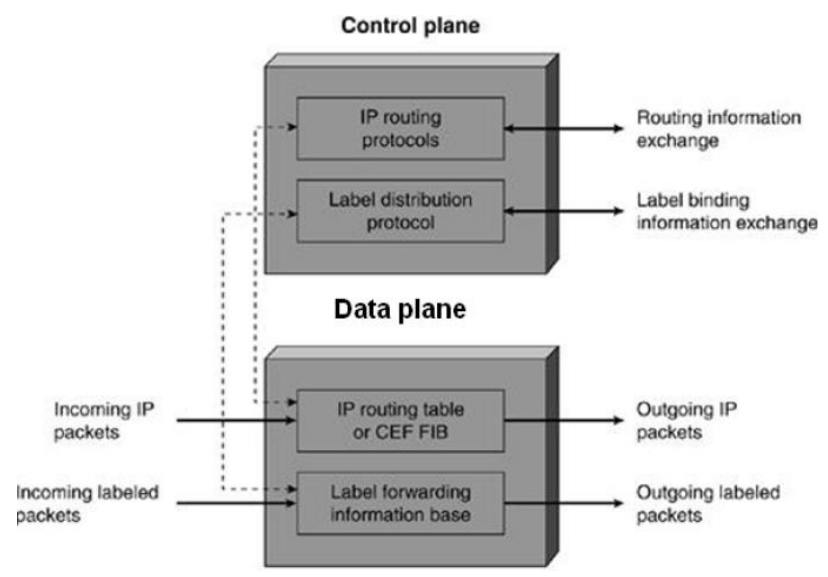

Figure 3: MPLS Architecture [3]

The control plane is responsible for the routing information exchanges and the label information exchanges with the adjacent routers. Link state routing protocols advertise routing information among the routers that are not necessarily adjacent, where-as label binding information distribution is limited to adjacent routers. The MPLS data plane has a simple forwarding engine, based on the information attached with labels. There are two tables on each MPLS router, LIB and LFIB. The data plane uses a label forwarding information base (LFIB) maintained by the MPLS enabled router to forward labelled packets. The LIB table contains all the local labels assigned by the local routers and mapping of the labels that it receives from the adjacent MPLS routers. The LFIB uses a subset of the labels contained in the LIB for actual packet forwarding. The MPLS enabled routers use 
information in LFIB and label value to make forwarding There are four major challenges in this network:

decisions.

The effective bandwidth for a system can be given by as follows:

$$
w_{i}^{e f f}\left(n_{i}\right)=\frac{\left(\alpha b(1-\rho)+\rho n_{i} d\right) r n_{i}}{\alpha b(1-\rho)+d n_{i}}
$$

The source sends its data to the destination. In MPLS domain, not all of the source traffic is necessarily transported through the same path. Depending on the traffic characteristics, different LSPs could be created for packet with different CoS (Class of Service) requirement. MPLS label is inserted between layer 2 and layer 3 and is 32 bit long. Figure shows the MPLS routing process in larger networks. There are two types of routers, edge routers and core routers. The routing decisions are made only at the edge routers and the core routers forward packets based on the labels.

High QoS requirement is one of the major issues for network service providers. The main QoS parameters are bandwidth optimization, low packet loss ratio, low hop count and low link load etc. For this, it introduces the concept of fast re-routing to bind the restoration latency in MPLS networks. This routing algorithm computed primary and backup paths to optimize the restoration latency and the amount of bandwidth used.

QoS is the mechanism of the network to provide different service level to a different traffic type as business need. Service providers offer their network service with quality. They define a Service-Level Agreements (SLA). SLA provides the details of all QoS parameters. It defines the parameters such as end-to-end delay, end-to-end jitter, packet loss. QoS is not single device functionality and it is an end to end mechanism. It provides the intelligence to network devices to treat the different application's traffic as their defined service level by SLA.

QoS combines different technologies together such as classification, marking, scheduling, queuing, bandwidth allocation, and prioritization that are commonly used to provide a scalable end to end service. QoS is a generic term. It provides the different level of treatment to the different types of traffic or applications that flows over network. Quality of service is required to provide the well management of network resources that makes the sophisticated usage of resources and gives comfort to network user. Business networks are widely expended with different types of applications. These applications have different network requirements. It needs to lead for different administrative policies that control applications as per their requirements individually. QoS within a network is essential to meet the requirements of today's converged networks. QoS provides the different levels of service for business critical application and delay-sensitive applications.

QoS is to manage the following network elements:

- Bandwidth: Maximum amount of data that can be carried.

- Delay: The time to send data from source to destination.

- Jitter: Variation in delay.

- Reliability: Packet loss.
- Bandwidth

- Delay

- Jitter

- Packet loss

- Fault tolerance

All the mentioned QoS parameters are explained below:

1. Bandwidth

The amount of data that can be transmitted over link is bandwidth. On the network IP Packets travel though the best route. Maximum bandwidth of the route is equal to smallest value of bandwidth on route.

\section{Delay}

End to end delay is the total time that a packet takes from source to destination.

End-to-end delay is sum of all the following delays.

- Processing delay

- Queuing delay

- Serialization delay

- Propagation delay

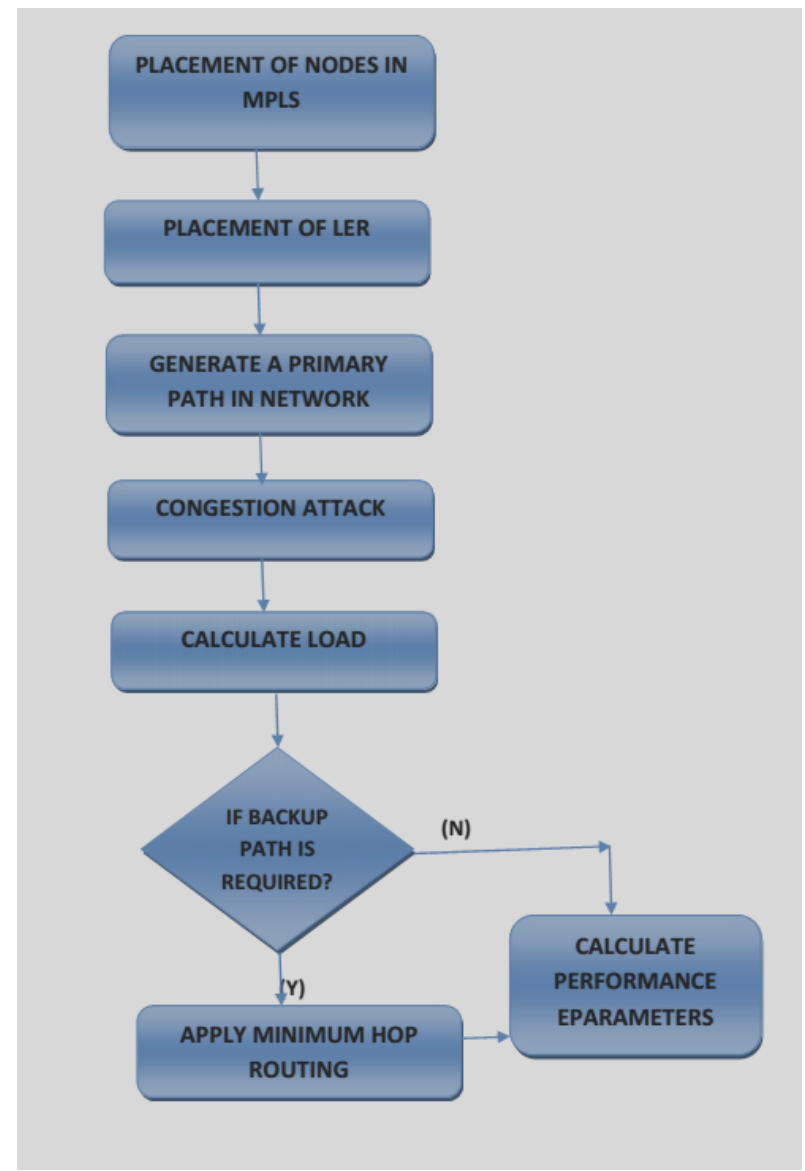

Figure 4: Proposed Steps of System

3. Jitter

Variation in delay is jitter. Packets for the same destination may not arrive at same rate. Campus network run different applications simultaneously. Jitter can occur due to different traffic load on different timings. For voice and video it is necessary to receive the packets at same sequence to achieve good quality. 


\section{Packet Loss}

Packet loss occurs due to the low buffer space. When the buffers space of the interface full then packets are dropped. Packet loss creates extended delays and jitter. Packet loss can be controlled by applying some techniques such as Tail Drop, Random Early Detection, Weighted Random Early Detection and Traffic Shaping and Policing.

There are many advantages to using MPLS. It enables a single converged network to support both new and legacy services, allowing efficient migration to an IP-based infrastructure. MPLS operates over legacy infrastructures such as SONET and new infrastructures (Ethernet) and networks (IP, ATM, Frame Relay, Ethernet and TDM).

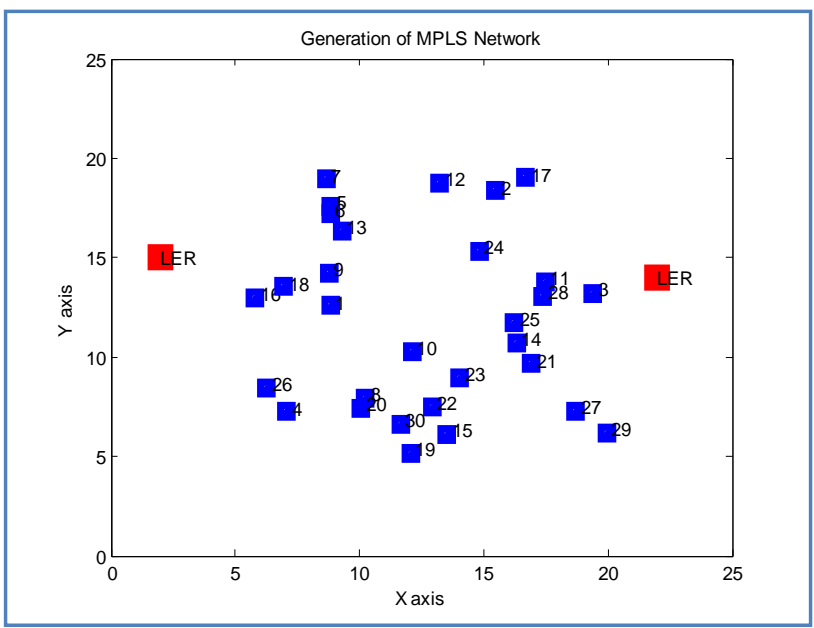

Figure 5: Generation of MPLS Network using MATLAB

The word "Multiprotocol" indicates that MPLS has the ability to carry multiple network protocols. Another advantage of MPLS is that it does not require high degrees of router processing from the label-switch routers for the forwarding since the most intensive part of the process, which is the assignment decisions, has been made at the label edge routers. Less high-end routers and switches can be used to perform the forwarding instead.

\section{RESULTS \& DISCUSSION}

MPLS integrates the performance and traffic management of level layer 2 (L2) with the scalability and flexibility of routing on layer 3 (L3). Therefore, it is seen as the protocol of L2.5 level. Conventional routing is based on the exchange of the information about the availability of the network, as packet travels through the network; each router extracts the information relevant to forwarding from L3 headers. This information is then used to index the routing tables to determine the next hop for the packet. This is repeated at each router in the network. At each hop in the network, the optimal forwarding packets must be reestablished.

The main concept of MPLS is to add labels in each packet. Based on these labels the packet forwarding through the network is done. However, the label summarizes essential information for routing the packet through MPLS domain. Hence, MPLS is a technology that accelerates and directs the flow of network traffic and makes it easier to manage. As we know that better QoS is the main priority for the network service providers. As MPLS is a differentiated and scalable framework which can provide effective bandwidth requested for any application. So we have designed such a model which can fulfil the entire requirement needed by network consumers.

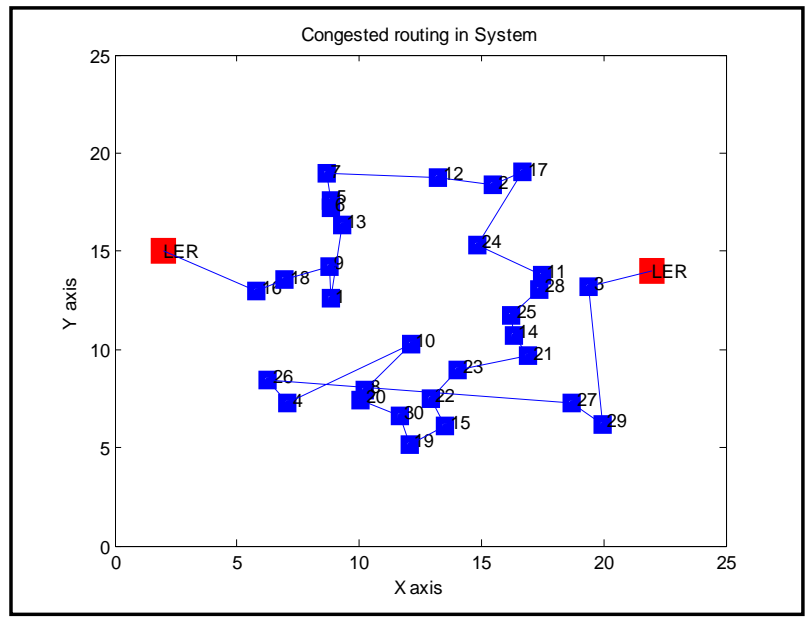

Figure 6: Congested Routing in MPLS Network

The network consists of 30 nodes. All links were setup as duplex with some initial delay and using Drop Queuing, which serve packets on a First Come First Serve (FCFS) basis.

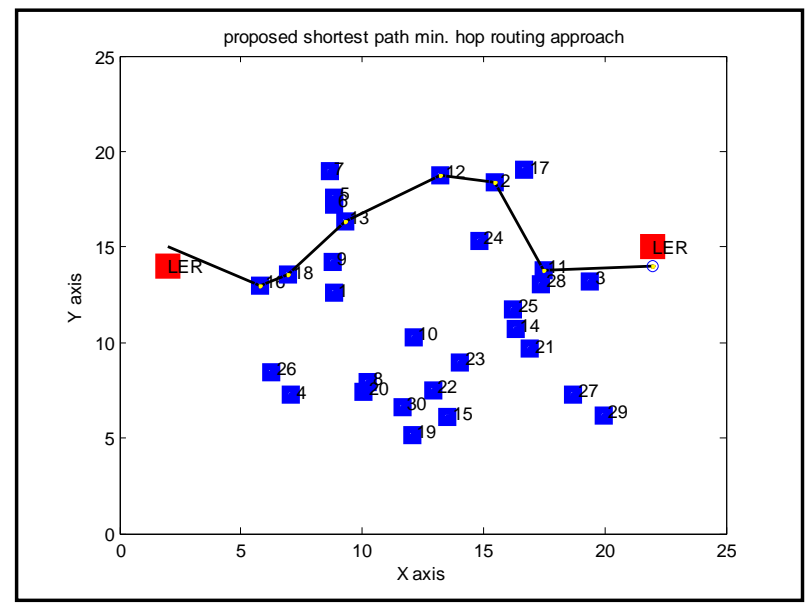

Figure 7: Improved Re-Routing in MPLS Network

The shortest possible path in a network with n nodes is one hop; the longest possible path (without loops) is (n-1) hops. The number of packets that belong to a particular flow and traverse the network at one time depends on packet inter arrival times and network latency. To estimate the impact of changes to topologies, it is assumed that propagation and transmission delays are the same for all links. The shortest path algorithm calculates the shortest path using the number of routers as the cost function. Dijkstra's algorithm is called the single-source shortest path. It is also known as the single source shortest path problem. It computes length of the shortest path from the source to each of the remaining vertices in the graph. Dijkstra's algorithm uses the greedy approach to solve the single source shortest problem. They provide communication by minimum hop routing technique. Information is securely 
transferred from sender to the receiver. It selected the shortest path from sender to receiver as shown in fig 7.

In figure 8 , effect of queuing delay on bandwidth has shown with the help of simulation results. From the results shown below we can conclude that for the same number of traffic volume, bandwidth required is more in case when queuing delay is less. It is one of the major factors which limit the performance of any service providing network. So significant improvement in bandwidth is done by varying this parameter.

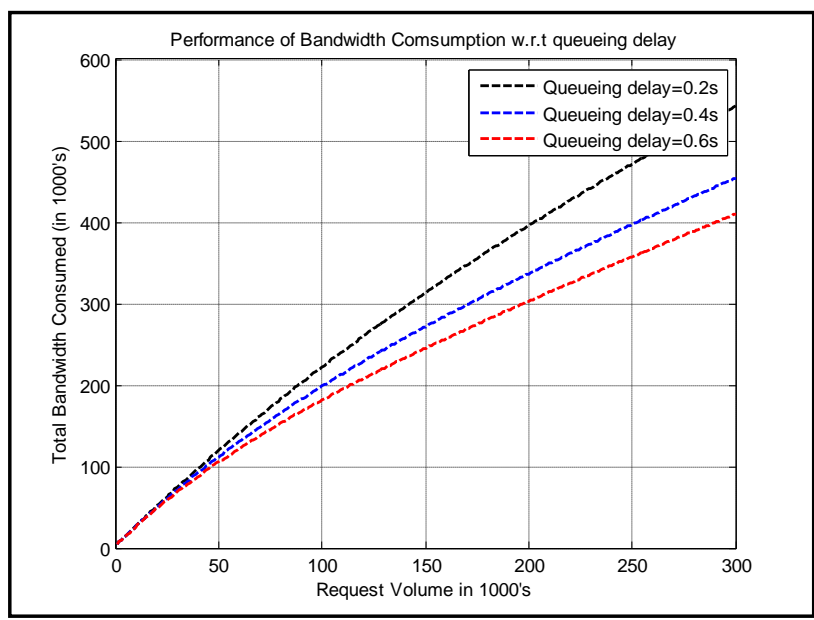

Figure 8: Performance of Bandwidth Consumption w.r.t Delay

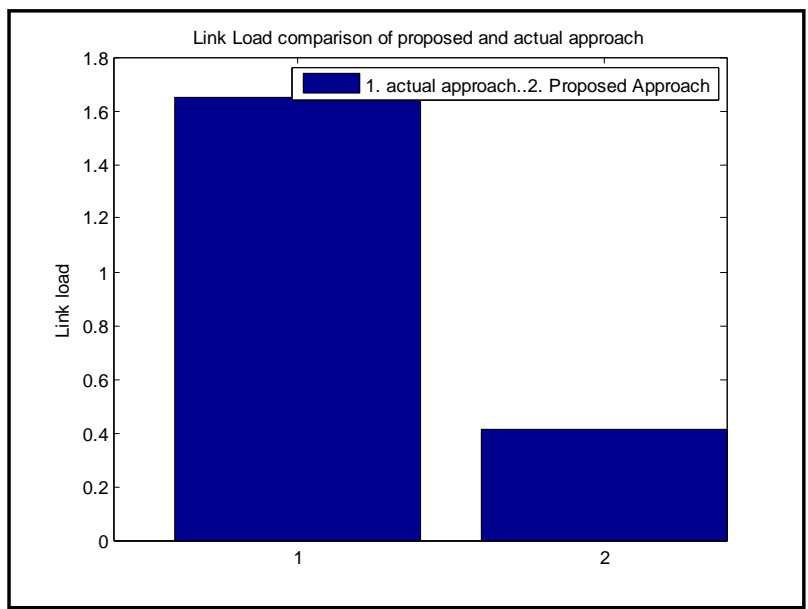

Figure 9: Performance of MPLS Network in terms of Link Load Parameter

\section{CONCLUSION}

MPLS has been developed for supporting traffic engineering and quality-of-service (QoS) guarantees in Internet backbone networks. These networks provide connection-oriented data transfer services based on label switched paths (LSPs) established between label edges router (LER) pairs. In this work, it presents a Rerouting model in MPLS Network for reducing traffic. The efficient QoS model provides better control and administration of network traffic. Solution of routing problem with help of proposed model allows providing the distribution of traffic between source- and destination-node so that delays along every path are equal between each other. Depending on the parameters of the model it is possible to implement different schemes of reservation: link, node or path protection. The results show the improvement in bandwidth as well as throughput. The performance of bandwidth consumption is shown w.r.t delay produced. The link load of proposed approach is also better.

\section{REFFERENCES}

[1] Ivana Hucková, Martin Hrubý, "QoS-Based Optimization of Data Flow in MPLS Networks", IEEE 2015.

[2] Olexandr Lemeshko, Kinan Arous, "Fast Re Route Model for Different Backup Schemes in MPLS-Network", IEEE First International Scientific-Practical Conference, 2014.

[3] Azeddien M. Sllame, "Modeling and Simulating MPLS Networks", IEEE 2014.

[4] Olexandr Lemeshko, Tatiana Vavenko, "Design of Multipath routing Scheme with Load Balancing in MPLS-network", IEEE 2013.

[5] Jorge Crichigno, Joud Khoury, "Routing in MPLS Networks with Probabilistic Failures", IEEE Communication QoS, Reliability and Modeling Symposium, 2013.

[6] Abdelnour Aldraho, Alexander A. Kist, "Performance Investigation of Dynamic Topologies in MPLS Networks", IEEE Performance Investigation of Dynamic Topologies in MPLS Networks, 2013.

[7] Chin-Ling Chen, "A Proposal of Next Generation Network: QoS Mapping for MPLS-DiffServ and Label Forwarding", IEEE 5th International Conference on BioMedical Engineering and Informatics, 2012.

[8] Anuar Zamani Othman, Ruhani Ab Rahman, "The Effect of QoS Implementation in MPLS Network", IEEE Symposium on Wireless Technology and Applications, 2012

[9] A. Kvalbein and O. Lysne. How can multi-topology routing be used for intradomain traffic engineering? Pages 280-284. ACM, 2007.

[10] Amund Kvalbein, Audun Fosselie Hansen, Tarik Cicic, Stein Gjessing, and Olav Lysne. Multiple routing configurations for fast ip network recovery. IEEElACM Trans. Netw., 17:473-486, April 2009

[11] Ye Wang, Hao Wang, Ajay Mahirnkar, Richard Alimi, Yin Zhang, Lili Qiu, and Yang Richard Yang. R3: resilient routing reconfiguration. SIGCOMM Comput. Commun. Rev., 40:291302, August 2010.

[12] Abdelnour Aldraho and Alexander A. Kist. Enabling dynamic topologies in communication networks. 2011 Australasian Telecommunication Networks and Applications Conference (ATNAC 2011), 2011.

[13] Mike Bennett, Ken Christensen, and Bruce Nordman. Improving the energy efficiency of ethernet: Adaptive link rate proposal. Technical report, ethernet alliance, Austin, USA,J uly 2006.

[14] Rugged Com Inc. Latency on a switched ethernet network, April 2008.

[15] Jerry D. Gibson, Bo Wei, and Sayantan Choudhury. Voice communications over tandem wire line IP and WLAN connections. 2006.

[16] Voice over IP - per call bandwidth consumption. Cisco Systems, 2006. 